\title{
Biofilm fúngico asociado a dispositivo intravascular de larga permanencia: Complicaciones y dificultades terapéuticas
}

\author{
Giancarlo Pérez-Lazo¹, Julio Maquera-Afaray², Renzo Soca ${ }^{3}$, Kevin Pacheco-Barrios ${ }^{4}$, Raúl Castillo-Córdova ${ }^{1}$ \\ RESUMEN
}

Las infecciones fúngicas asociadas a biofilms en dispositivos biomédicos son refractarias al tratamiento antifúngico y habitualmente se requiere del retiro oportuno del dispositivo, así como la administración de antifúngicos sistémicos. Presentamos el caso de una paciente mujer de 36 años que recibe terapia para el dolor administrada por dispositivo intravascular de larga permanencia, catéter Port-A-Cath, y que desarrolló candidemia y endocarditis por Candida parapsilosis asociada al catéter; el cual tuvo que ser retirado mediante toracotomía por persistencia de la infección a pesar de la terapia antifúngica sistémica. Se evidenció extenso biofilm rodeando al catéter, con aislamiento de Candida parapsilosis y se completaron seis semanas de tratamiento antifúngico con evolución clínica favorable.

Palabras clave: Biopelícula; candidemia; catéter intravascular de larga permanencia (Fuente: DeCS BIREME).

\section{Fungal biofilm associated with long-term central venous access device: Complications and therapeutic difficulties}

\section{ABSTRACT}

Fungal infections associated with biofilms in biomedical devices are refractory to antifungal treatment and usually require the timely removal of the device, as well as the administration of systemic antifungals. We present the case of a 36-yearold female patient who received pain therapy administered by a Port-A-Cath long-term central venous access device. She developed catheter-related candidemi a and endocarditis caused by Candida parapsilosis. The catheter had to be removed by thoracotomy due to persistent infection despite the systemic antifungal therapy. An extensive biofilm surrounding the catheter was observed and Candida parapsilosis was isolated. Six weeks of antifungal treatment with a favorable clinical evolution were completed.

Keywords: Biofilm; candidemia; long-term central venous access catheter (Source: MeSH NLM).

1. Servicio de Medicina Interna, Infectología, Hospital Guillermo. Almenara Irigoyen, EsSalud. Lima, Perú.

2. Escuela de medicina, Universidad Privada de Tacna. Tacna, Perú.

3. Departamento de Cardiología, Instituto Nacional Cardiovascular. Lima, Perú.

4. Laboratorio de Neurociencia y Comportamiento-Universidad Peruana Cayetano Heredia. Lima, Perú. 


\section{INTRODUCCIÓN}

Desde 1982, se han incorporado los reservorios venosos centrales totalmente implantables, que comprenden un puerto implantado subcutáneamente (o reservorio) conectado a un catéter venoso central, más frecuentemente insertado en la vena yugular interna, subclavia o cefálica; por el cual se pueden administrar quimioterapia antineoplásica, nutrición parenteral, productos sanguíneos, tratamiento antimicrobiano prolongado y analgesia ${ }^{(1,2,3)}$. Este tipo de catéteres fueron desarrollados para reducir el riesgo de efectos tóxicos y de colonización bacteriana o fúngica mediante el uso de una ruta subcutánea o tunelizada que impide la migración de microorganismos presentes en la superficie cutánea, sin embargo la complicación más frecuente asociada es la infección (2), lo cual constituye una dificultad terapéutica debido a la presencia de biopelículas o también conocidas como biofilm, que se definen como una comunidad de microorganismos adheridos a una superficie, y rodeados de una matriz extracelular ${ }^{(4,5)}$. Aunque los biofilms bacterianos han sido más estudiadas, las especies de Candida son organismos patógenos que cada vez tienen mayor relevancia en este tipo de infecciones ${ }^{(5)}$.

Presentamos el caso de una paciente portadora de un dispositivo implantable permanente, tipo Port-A-Cath (PAC), que desarrolló candidemia y endocarditis por Candida parapsilosis asociada al dispositivo intravascular con producción de biofilm fúngico.

\section{CASO CLÍNICO}

Paciente mujer de 36 años, con diagnóstico de vasculitis leucocitoclástica y uso de morfina como terapia para el dolor crónico, administrada por PAC desde hace un año, sin antecedente de episodios previos de infección del catéter. Ingresa por emergencia por 10 días de fiebre intermitente, tos y dolor torácico, con evidencia tomográfica de infiltrado pulmonar en base de hemitórax derecho y se decidió iniciar cobertura antibiótica de amplio espectro con imipenem y vancomicina. Al examen físico no se evidenció signos inflamatorios en zona externa del catéter. En el quinto día de hospitalización se reportó aislamiento de C. parapsilosis, en un set de hemocultivos periféricos tomados al ingreso, con susceptibilidad a fluconazol $(C I M \leq 2 \mu \mathrm{g} / \mathrm{mL})$, caspofungina $(C I M \leq 2 \mu \mathrm{g} / \mathrm{mL})$, anidulafungina (CIM $\leq 2 \mu \mathrm{g} / \mathrm{mL})$ y voriconazol (CIM $\leq 0,12 \mu \mathrm{g}$ / $\mathrm{mL}$ ), según antifungigrama (método automatizado Vitek2puntos de corte aprobados CLSI/M27-S4-2012). La paciente refirió exposición previa a azoles. Inició tratamiento con caspofungina 70mg EV dosis de carga y luego 50mg EV c/24 horas por 5 días; y con hemocultivos posteriores negativos a las 48 horas más caída de la fiebre, se decide continuar tratamiento con fluconazol $800 \mathrm{mg} /$ día EV hasta completar 14 días de tratamiento antifúngico. El examen de fondo de ojo fue normal, y por la aparente evolución favorable no se realizó estudio ecocardiográfico. Sin embargo, a los dos días de haber concluido la terapia antifúngica cursó con deterioro clínico evidenciándose en nueva tomografía de tórax imagen sugestiva de trombo en el extremo distal de la arteria pulmonar derecha con extensión a ramas interlobares (Figura 1), lo cual coincide con nuevo cuadro febril y dolor torácico, por lo que se realizó inmediatamente una ecocardiografía transesofágica (ETE), donde se objetivó en el PAC múltiples vegetaciones > 20 $\mathrm{mm}$ en su trayecto, siendo la mayor de $4 \mathrm{~cm}$ (Figura 2).

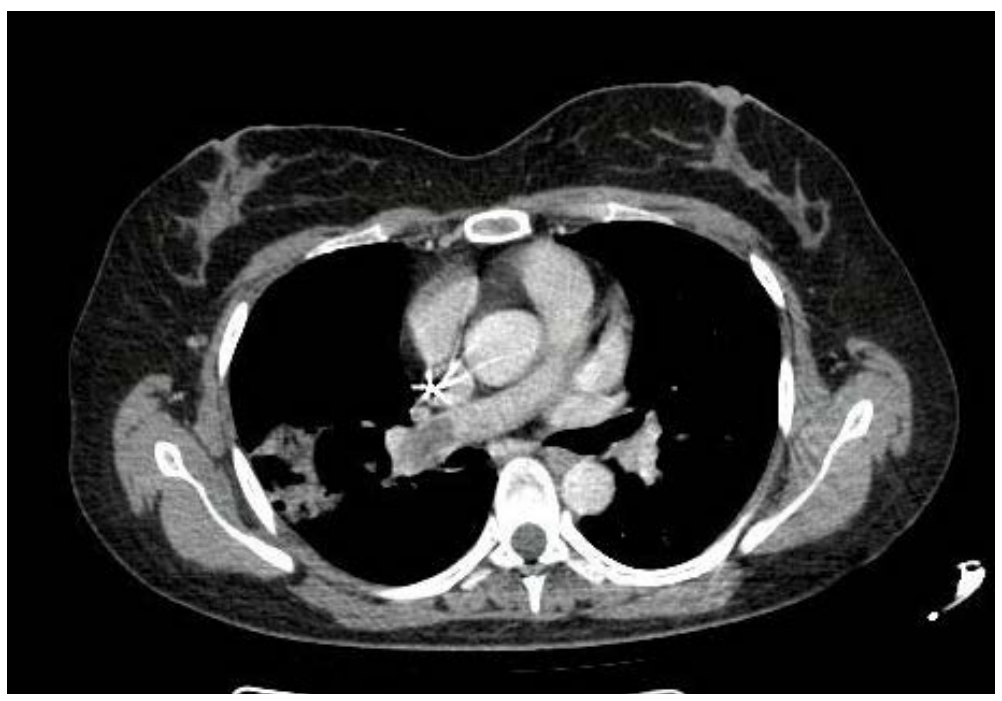

Figura 1. Tomografía espiral multicorte ( TEM) tórax : Imagen hipodensa en extremo distal de arteria pulmonar derecha sugerente de trombo a ese nivel 


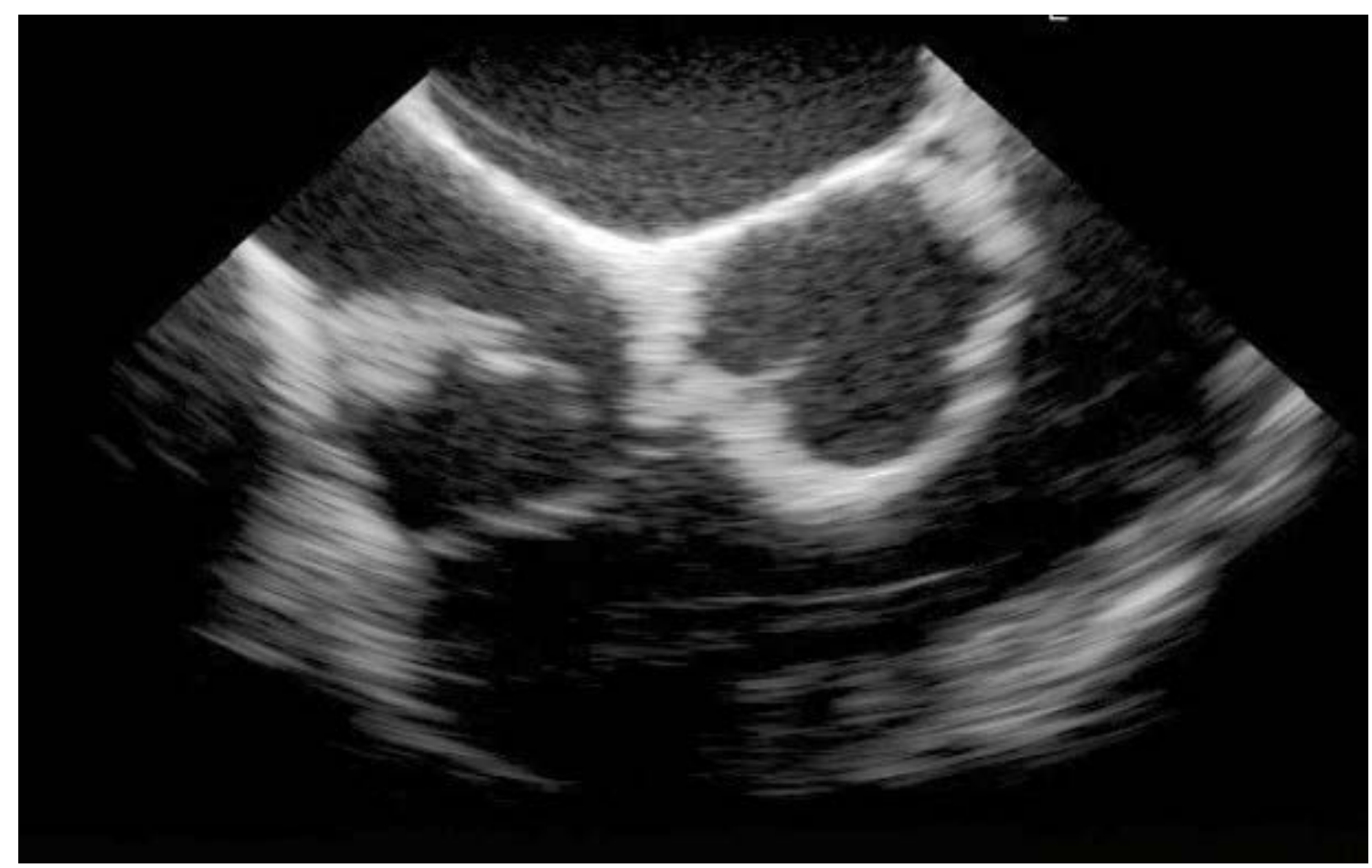

Figura 2. Ecocardiografía transesofágica (ETE) que muestra Port-A-Cath (PAC) con recorrido aurículo-ventricular derecho, y múltiples vegetaciones $>20 \mathrm{~mm}$ en su trayecto, siendo la mayor de $4 \mathrm{~cm}$

Inició cobertura empírica para endocarditis con imipenem y vancomicina, con resultados de hemocultivos periféricos negativos. No se tomaron muestras de la punta ni del septum subcutáneo. Por la sospecha de endocarditis fúngica, se decide retiro del dispositivo intravascular mediante toracotomía, siendo realizado a las 48 horas del inicio de antibióticos, objetivándose la presencia de importante biofilm (Figura 3), con resultado de cultivo directo de la pieza extraída que reportaba C. parapsilosis (Figura 4). La susceptibilidad de la cepa fue la misma que el episodio previo de candidemia y se dirigió la cobertura antifúngica con caspofungina 70mg EV dosis de carga y luego completó
30 días con 50mg EV/día, posterior al retiro del PAC y negativización de hemocultivos, continuando con fluconazol $800 \mathrm{mg} /$ día por 2 semanas adicionales.

La evolución clínica fue favorable y la fiebre cedió tras retiro del PAC. La ETE realizada a las 2 semanas de tratamiento antifúngico no evidenció vegetaciones ni abscesos perivalvulares y en tomografía de tórax control se evidenció mejoría de consolidado basal derecho inicial sin imágenes embólicas. La paciente llega a manejar el dolor con tratamiento vía oral, siendo dada de alta. 


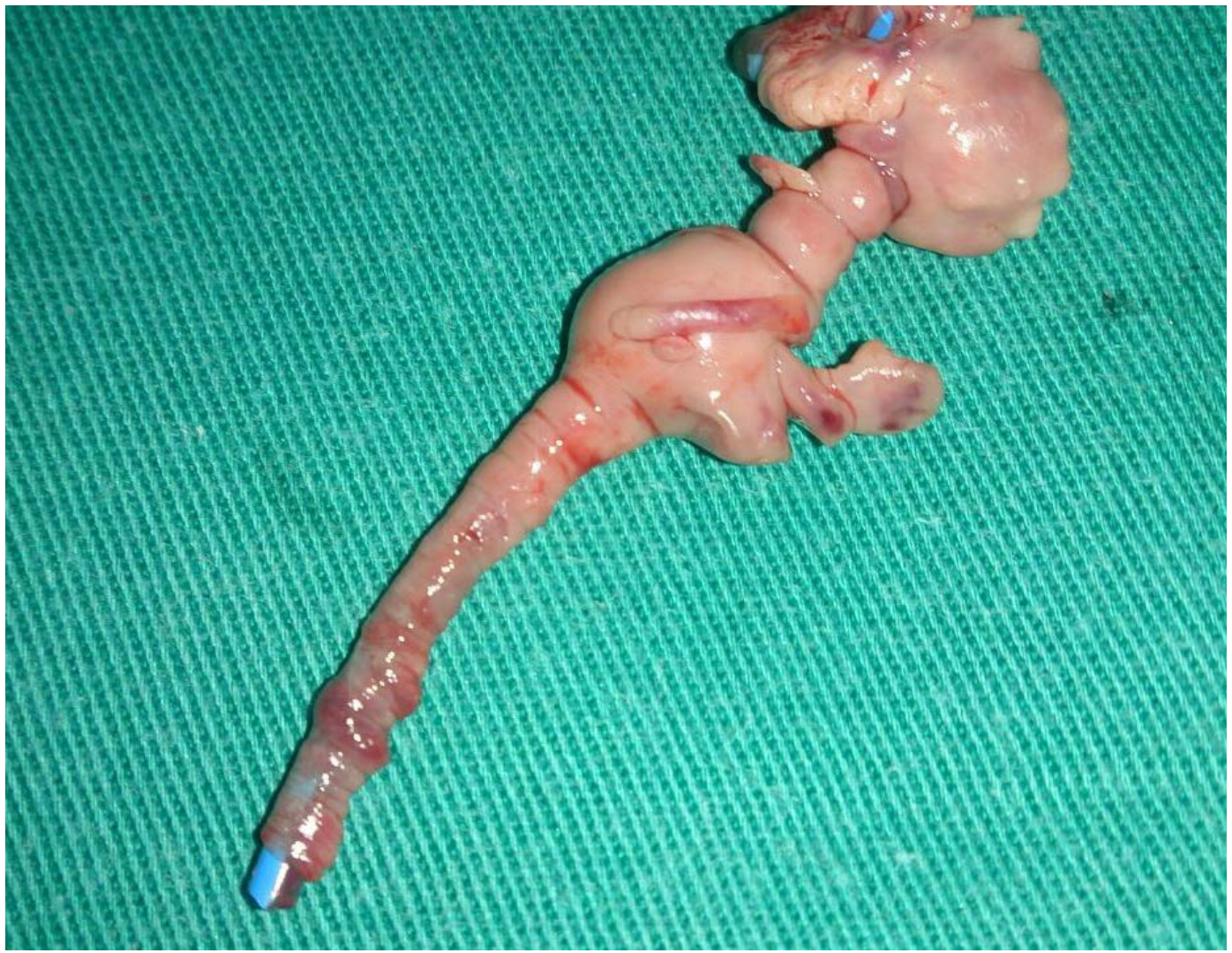

Figura 3. Anatomía macroscópica: Port-A-Cath (PAC) que evidencia múltiples vegetaciones adheridas en su superficie

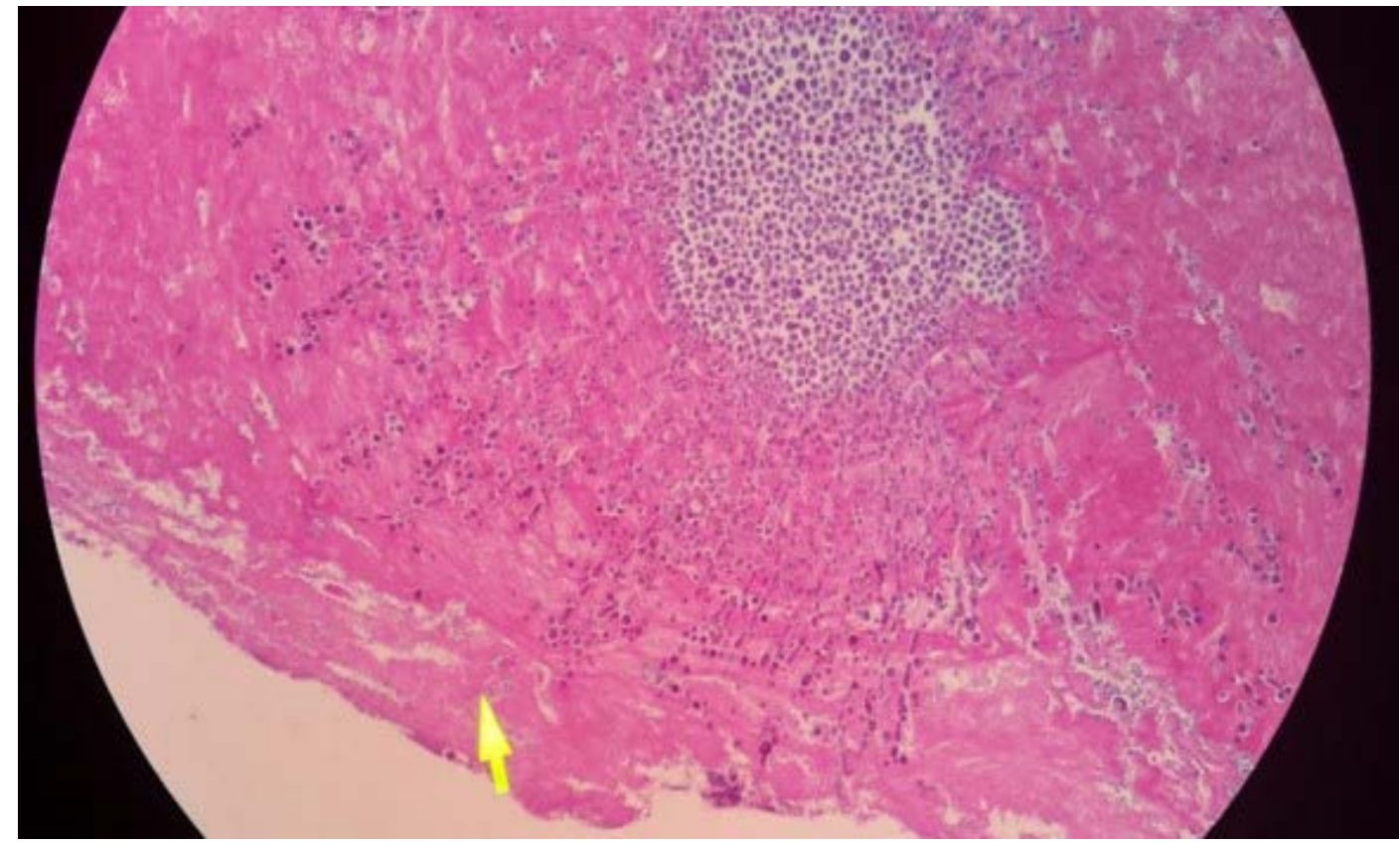

Figura 4. Microscopía de pieza extraída. Se observan la presencia de levaduras y pseudohifas. Tinción de hematoxilina y eosina (10x) 


\section{DISCUSIÓN}

Las especies de Candida son capaces de colonizar los reservorios vasculares implantables y formar biofilms (5). Los biofilms se desarrollan cuando los organismos se adhieren a una superficie y producen polímeros extracelulares que proveen una matriz extracelular que facilita la adhesión posterior ${ }^{(6)}$. Esta matriz está formada por polisacáridos, hidratos de carbono, proteínas, ácidos nucleicos, fosfatos y ácido úrico producidos por las células que forman el biofilm; además, es altamente hidratada (98\% de agua), confiriéndole un aspecto viscoso y gelatinoso ${ }^{(4,5)}$.

Es importante destacar que la matriz extracelular de los biofilms dificulta la entrada de los antimicrobianos (grado y velocidad de penetración) ${ }^{(4,5)}$, siendo la presencia de biofilm en nuestra paciente la causa de recurrencia de la infección tras cese de la terapia antifúngica. También se debe considerar en estos casos la posibilidad de resistencia a los antifúngicos usados (7); sin embargo, la resistencia a fluconazol en caso de C. parapsilosis está reportada entre 0 a $4.6 \%{ }^{(8)}$, y en el estudio peruano de Rodríguez et al. se encontraron sólo 2 cepas de $C$. parapsilosis resistentes a fluconazol (9). En el caso presentado se evidenció susceptibilidad a azoles y equinocandinas, pero la exposición previa a fluconazol pudo favorecer la resistencia o aparición de una cepa con susceptibilidad disminuida a azoles ${ }^{\left({ }^{8}\right)}$.

En el tratamiento de biofilms de Candida, han demostrado cierta eficacia las equinocandinas o la anfotericina $B$ liposomal ${ }^{(5)}$. Se postula que la liberación de 1,3 B-glucano en la matriz extracelular tendría un rol en disminuir la susceptibilidad de los antifúngicos no relacionados con la inhibición de este componente; por ello, existe marcada tolerancia a fluconazol y anfotericina en biofilms de C. albicans, asociados a cambios en la arquitectura por el incremento de 1,3 B-glucano y secuestro de estos agentes (10,11). Nosotros utilizamos caspofungina en el tratamiento inicial del primer episodio de candidemia, así como en el tratamiento dirigido para la endocarditis por el biofilm de Candida parapsilosis, sin embargo, un asunto a resaltar es el perfil de susceptibilidad de esta cepa a las equinocandinas; pues tiene la presencia de un polimorfismo natural en los puntos calientes de mutación (hotspot region) del gen FKS1, llevando a unos mayores valores en la concentración inhibitoria mínima (CIM) de estos agentes en comparación a otras especies de Candida. Este hallazgo ha llevado al debate si las equinocandinas pueden ser consideradas para tratar candidiasis invasivas por C. parapsilosis. En un subanálisis del estudio multicéntrico en 29 hospitales de España, Férnandez-Ruiz et al. demostraron que el inicio de equinocandinas no tiene efectos negativos en los resultados del manejo de fungemia por $C$. parapsilosis, pero destacan el factor protector del retiro temprano del catéter venoso central ${ }^{(12)}$. Consideramos este hecho determinante en la evolución favorable de nuestra paciente, recomendado en las guías actuales sobre manejo de candidemias asociadas a catéter ${ }^{(13,14)}$. No obstante, en la práctica clínica, el retiro de catéter no siempre es fácil de realizar. El retiro de catéteres totalmente implantados, como el PAC requiere de un procedimiento quirúrgico. Más aún, el estado clínico del paciente puede afectar la decisión del clínico y no debe ser considerada posible para pacientes deteriorados con trombocitopenia severa, coagulación intravascular diseminada o problemas anestésicos ${ }^{(8)}$.

Como estrategia adicional para la prevención y erradicación de biofilms fúngicos por Candida se incluye: "lock therapy", que consiste en verter altas dosis de equinocandinas y anfotericina B liposomal en gotas, directamente en el catéter y su posterior bloqueo en el interior por un tiempo (desde varias horas a días), asociado a terapia antifúngica sistémica. En la mayoría de los casos clínicos reportados, este tipo de tratamiento fue administrado luego de que la terapia sistémica fallase, pero con riesgo de toxicidad asociada y generación de resistencia ${ }^{(5)}$.

En conclusión, este reporte de caso de biofilm fúngico nos permite conocer ciertas consideraciones terapéuticas de importancia, dado el aumento del uso de dispositivos intravasculares totalmente implantables en los últimos años; pues este tipo de infecciones son refractarias a los antifúngicos y se requiere en muchas ocasiones el retiro del dispositivo intravascular como el caso presentado. Además, debemos considerar las limitaciones en hospitales peruanos para el acceso de antifúngicos idóneos en caso de biofilms, como las equinocandinas o formas liposomales de anfotericina.

\section{REFERENCIAS BIBLIOGRÁFICAS}

1. Yousif A., Jamal M.A., Raad I. (2015) Biofilm-Based Central Line-Associated Bloodstream Infections. In: Donelli G. (eds) Biofilm-based Healthcare-associated Infections. Advances in Experimental Medicine and Biology, vol 830. Springer, Cham. DOI: 10.1007/978-3-319-11038-7_10.

2. Lebeaux D, Fernández-Hidalgo N, Chauhan A, Lee S, Ghigo J, et al. Management of infections related to totally implantable venous-access ports: challenges and perspectives. Lancet Infect Dis. 2014 Feb;14(2):146-59.

3. Biffi R. (2012) History of Vascular Access. In: Di Carlo I., Biffi R. (eds) Totally Implantable Venous Access Devices. Springer, 
Milano. DOI: 10.1007/978-88-470-2373-4_1.

4. Pozo JL, Cantón E. Candidiasis asociada a biopelículas. Rev Iberoam Micol 2016; 33:176-83.

5. Bujdáková H. Management of Candida biofilms: state of knowledge and new options for prevention and eradication.. Future Microbiol. 2016;11(2):235-51.

6. Kojic EM, Darouiche RO. Candida Infections of Medical Devices. Clinical Microbiology Reviews. 2004;17(2):255-267.

7. Høiby et al. ESCMID Biofilm guideline. Clin Microbiol Infect 2015; 21: S1-S25.

8. Devrim I, et al. A Single Center's Experience with Candida parapsilosis Related Long-Term Central Venous Access Device Infections: The Port Removal Decision and Its Outcomes. Pediatr Hematol Oncol. 2014;31(5):435-41.

9. Rodriguez L, Bustamante B, Huaroto L, Agurto C, Illescas R, Ramirez R, et al. A multi-centric Study of Candida bloodstream infection in Lima-Callao, Peru: Species distribution, antifungal resistance and clinical outcomes. PLoS ONE 2017,12(4): e0175172.

10. Hirota K, Yumoto H, Sapaar B, Matsuo T, Ichikawa T, Miyake, Y. Pathogenic factors in Candida biofilm-related infectious diseases. J Appl Microbiol 2017; 122: 321-330.

11. Nett J, Lincoln L, Marchillo K et al. Putative role of beta-1,3 glucans in Candida albicans biofilm resistance. Antimicrob. Agents Chemother. 2007;51(2), 510-520.

12. Fernández-Ruiz et al. Initial Use of Echinocandins Does Not Negatively Influence Outcome in Candida parapsilosis Bloodstream Infection: A Propensity Score Analysis. Clinical Infectious Diseases 2014;58(10):1413-21

13. Pappas P, Kauffman C, Andes D, Clancy C, Marr K, OstroskyZeichner L, et al. Clinical Practice Guideline for the Management of Candidiasis: 2016 Update by the Infectious Diseases Society of America. Clin Infect Dis 2016; 62 (4): e1e50.
14. Cuenca-Estrella et al. ESCMID* guideline for the diagnosis and management of Candida diseases 2012: diagnostic procedures. Clin Microbiol Infect 2012; 18(7): 9-18

Fuentes de financiamiento:

Este artículo ha sido financiado por los autores.

Conflictos de interés:

Los autores declaran no tener ningún conflicto de interés.

\section{Correspondencia:}

Giancarlo Pérez Lazo

Dirección: Av. Grau Nro. 800. Lima 13, Perú.

Teléfono: 987113652

Correo electrónico: diamantdust@hotmail.com

Recibido: 08 de noviembre de 2017

Evaluado: 10 de noviembre de 2017

Aprobado: 21 de marzo de 2018

(c) La revista. Publicado por Universidad de San Martín de Porres, Perú. (c) в bajo términos de Licencia Creative Commons Atribución 4.0 Internacional. (http://creativecommons.org/licenses/by/4.0/)

\section{ORCID iDs}

Giancarlo Pérez Lazo Julio Maquera Afaray Renzo Soca Meza Kevin Pacheco Barrios Raúl Castillo Córdova https://orcid.org/0000-0003-3823-4737 https://orcid.org/0000-0002-3698-8343 https://orcid.org/0000-0002-1018-6764 https://orcid.org/0000-0002-7166-2816 https://orcid.org/0000-0001-6316-3331 